REGARDS

SUR L'ECONOMIE ALLEMANDE

BULLETIN ECONOMIQUE DU CRAC

\section{Regards sur l'économie allemande}

Bulletin économique du CIRAC

$68 \mid 2004$

Varia

\title{
Une économie en transition
}

Isabelle Bourgeois

\section{(2) OpenEdition}

Journals

Édition électronique

URL : http://journals.openedition.org/rea/3616

DOI : 10.4000/rea.3616

ISBN : 978-2-8218-0832-4

ISSN : 1965-0787

Éditeur

CIRAC

Édition imprimée

Date de publication : 1 octobre 2004

Pagination : 3-4

ISSN : 1156-8992

Référence électronique

Isabelle Bourgeois, "Une économie en transition », Regards sur l'économie allemande [En ligne], 68 | octobre 2004, mis en ligne le 24 juillet 2015, consulté le 15 septembre 2020. URL : http://

journals.openedition.org/rea/3616 


\section{Une économie en transition}

L'embellie modérée que le président de la BCE évoquait en septembre à propos de la zone euro vaut également pour l'Allemagne. Les taux de croissance des deux premiers trimestres de l'année sont pratiquement identiques, avec respectivement $+0,6 \%$ et $+0,5 \%$ dans la zone euro, $+0,4 \%$ et $+0,5 \%$ outre-Rhin. Pour l'ensemble de l'année, cela devrait impliquer un taux de l'ordre de $+2 \%$. Reste à savoir ce qu'il en sera réellement: jusqu'ici, la flambée du prix du pétrole n'a guère affecté les économies, mais si elle se poursuit, la croissance pourrait s'en ressentir. De même, le ralentissement de la dynamique américaine pourrait tasser les exportations, et ainsi affaiblir le principal moteur conjoncturel de la zone euro comme de l'Allemagne. Or dans les deux cas, la demande intérieure n'est pas assez solide pour prendre véritablement le relais. Cela est vrai surtout pour l'Allemagne à la consommation anémique, et dont la croissance pourrait dès lors se ralentir quelque peu vers la fin de l'année. Ces incertitudes expliquent pourquoi les prévisions de croissance pour 2004 varient actuellement entre +2,1 \% (RWI) et +1,5\% (HWWA).

Outre-Rhin, l'embellie du premier semestre résulte presque exclusivement de la très forte demande extérieure, surtout des pays tiers : en glissement annuel, les exportations ont augmenté de $+12,9 \%$ (IfW). Si les ventes se sont un peu ralenties vers la zone euro, la stabilisation du taux $€ / \$$ a relancé les exportations vers le marché américain. Quant à la Chine, elle occupe désormais la $10^{\mathrm{e}}$ place des pays destinataires, avec une très forte demande de produits dans les branches-phares de l'industrie allemande : construction mécanique (l'Allemagne est le premier exportateur mondial avec une part de marché de plus de $19 \%$ ), automobile, électrotechnique et informatique. L'industrie électronique et électrotechnique enregistrait au second trimestre une hausse de $+20,6 \%$ de ses exportations par rapport à la même période de l'an dernier ; la construction mécanique et l'industrie automobile, une augmentation de respectivement $+11,5 \%$ et $+11 \%$. Les carnets de commandes se remplissent donc, soutenant l'activité : la production industrielle a crû de $+2,7 \%$ au premier semestre 2004 par rapport au premier semestre 2003 (Destatis).

Les importations ont progressé à un niveau inférieur $(+7,6 \%$ en glissement annuel ; IfW), surtout celles de biens intermédiaires. Or cette hausse est moins générée par la demande intérieure (en léger recul au cours des six premiers mois) que par l'export, reflet d'un partage croissant du travail dans le contexte de la globalisation des activités. En effet, la part des importations entrant dans la production des biens exportés atteint aujourd'hui près de $40 \%$, soit dix points de plus qu'en 1995 (Destatis). Ce partage du travail, notamment avec les économies est-européennes, et particulièrement prononcé dans la filière automobile ou dans la construction mécanique, contribue à entretenir la compétitivité du « made in Germany » en permettant de calculer au plus juste coûts de production et, surtout, coûts salariaux. C'est ainsi que, depuis plusieurs années, la forte internationalisation de l'industrie tire la croissance globale d'un site national de production handicapé par ses coûts salariaux trop élevés. La multiplication des sites hors d'Allemagne est d'ailleurs l'une des principales raisons pour lesquelles les investissements d'équipement se maintiennent outre-Rhin à un faible niveau depuis quelque temps.

Or lorsque la concurrence mondiale s'avive, comme actuellement dans l'automobile ou l'électrotechnique, le handicap des coûts salariaux annexes devient un risque pour la compétitivité de ces branches et, partant, de l'économie allemande tout entière. C'est la raison pour laquelle, depuis l'été, se multiplient dans les entreprisesphares de ces secteurs les accords relevant la durée du travail afin de comprimer la masse salariale. Plus généralement, c'est dans la disproportion entre les coûts salariaux et la faible productivité horaire que réside la principale faiblesse structurelle de l'économie allemande. C'est elle qui explique pourquoi la croissance annuelle moyenne n'a été que de 1,4\% au cours de la décennie écoulée, affirme l'institut IfW de Kiel (Discussion Paper $\mathrm{n}^{\circ}$ 414). Au recul de la population en âge de travailler s'ajoute la baisse du volume des heures effectivement travaillées, passées à 1444 (Agence fédérale pour l'emploi). Quant à la Bundesbank, elle rappelle dans son rapport de septembre que, depuis 1991, les coûts salariaux horaires réels ont crû
La dynamique à l'export tire seule la conjoncture

Une industrie compétitive grâce à sa localisation mondialisée

Les coûts salariaux grèvent la compétitivité du site Allemagne 
Agenda 2010 : des effets bénéfiques à long terme

Etat-providence et unification à l'origine du déficit

Est : une économie aujourd'hui compétitive

La fin de l'approche égalitaire des politiques territoriales de $+1,8 \%$, alors que les salaires nets réels n'augmentaient, eux, que de $+0,7 \%$. Cela contribue aussi à expliquer la faible consommation des ménages allemands.

Ces constats ne peuvent que conforter dans sa politique le gouvernement Schröder dont l'Agenda 2010 cherche justement à agir sur la dérive du coût du système de protection sociale qui renchérit le coût du travail (la part des prélèvements dans les coûts salariaux dépasse $48 \%$ ) et qui génère un niveau incompressible de chômage structurel. Parallèlement, ses réformes du marché de l'emploi visent à accroître la population active occupée en incitant à la reprise d'un emploi. Ces mesures ne permettront pas de réduire immédiatement et significativement le nombre de chômeurs, qui devrait se maintenir aux alentours de 4,36 millions. Mais, conjuguées à la baisse des prélèvements et à la levée des 'rigidités' du marché de l'emploi à laquelle oeuvrent parallèlement les partenaires sociaux, elles finiront à terme par libérer la dynamique de croissance et générer de l'activité.

Cette même problématique creuse le déficit (3,7\% du PIB en 2004) et limite la marge de manœuvre budgétaire du gouvernement fédéral, dont la moitié des dépenses 2005 (48\%, soit 125 milliards $€$ ) est réservée au seul financement de la protection sociale. C'est là le tribut payé à un Etat-providence trop généreux, mais aussi à l'unité allemande : sur le total des aides versées par l'ouest depuis 1991 aux nouveaux Länder (1 250 milliards $€$ ), les trois quarts sont imputables aux transferts sociaux. Avec le vieillissement démographique, c'est l'autre origine du haut niveau des prélèvements outre-Rhin. Les aides réservées à la reconstruction de la structure économique des Länder de l'est sont faibles en comparaison. Et leur fin est programmée : le second Pacte de solidarité (Solidarpakt II), qui entre en vigueur en 2005 et porte sur un volume de 156 milliards €, expire à la fin 2019.

Il ne sera pas reconduit ; le processus de transformation est pratiquement achevé, constate le dernier rapport annuel sur l'état de l'unité allemande présenté en septembre par le gouvernement fédéral. En données CVS, au cours des dix dernières années, l'industrie est-allemande a enregistré une croissance moyenne annuelle de 5,5\%, son taux d'exportation est aujourd'hui de 24,5\%. Entre 1993 et 2002, le PIB des nouveaux Länder croissait de 3,7\% en moyenne annuelle selon une récente étude de la KFW ; mais la 'crise' du BTP masquait la réalité d'une réindustrialisation avancée, doublée de l'émergence de clusters particulièrement compétitifs. Le gouvernement fédéral réoriente donc sa politique structurelle vers une stratégie de soutien ciblé aux pôles de compétitivité.

Cette nouvelle approche de l'Aufbau Ost s'accompagne de l'acceptation de disparités territoriales. Dans une interview accordée au magazine Focus (13-09-04), le président de la République Horst Köhler rappelait que ces disparités sont une caractéristique foncière de l'Allemagne. Elles se reflètent dans le différentiel de croissance entre les Länder, mais aussi, au sein de chaque Land, entre les territoires. Ses propos ont déclenché une vive polémique, tant il est vrai qu'il hissait là dans l'espace public un thème hautement sensible : celui du principe constitutionnel de « l'équivalence des conditions de vie » que le Bund a pour mission de garantir sur l'ensemble du territoire (\$72.2 de la Loi fondamentale). Or le président fait ainsi écho au Tribunal constitutionnel fédéral qui, dans un arrêt du 11-11-1999 sur la péréquation financière entre les Länder, avait enjoint à ces derniers de réviser ce mécanisme de solidarité de manière à en respecter le principe sous-jacent : « l'interdiction du nivellement ». Et il avait fixé une date butoir : le 31-12-2004.

LA CROISSANCE NE S'AFFERMIRA DURABLEMENT en Allemagne que lorsque toutes les parties concernées auront engagé les réformes structurelles permettant de débrider la dynamique de croissance. Les partenaires sociaux commencent à lever les 'rigidités' du marché de l'emploi ; la réforme de l'Etat-providence est en cours ; mais l'Allemagne ne pourra respecter les critères de Maastricht que lorsque les Länder se plieront à la même discipline que le Bund et rompront avec la dérive égalitariste d'une solidarité fiscale et financière destinée, à l'origine, à garantir l'équité des chances à chaque collectivité et non à créer des conditions de vie uniformes sur l'ensemble du territoire. L'Allemagne respectera le Pacte de stabilité européen, ne serait-ce qu'au nom de sa propre dynamique de compétitivité future dans l'économie de la connaissance. Pour l'heure, son système économique et social est effectivement entré dans une phase de transition.

Isabelle Bourgeois (24-09-2004) 